Research

Open Access

\title{
Diagnostic utility of the soluble triggering receptor expressed on myeloid cells-1 in bronchoalveolar lavage fluid from patients with bilateral lung infiltrates
}

\author{
Jin Won Huh1, Chae-Man Lim², Younsuck Koh², Yeon Mok Oh², Tae Sun Shim², Sang Do Lee², \\ Woo Sung Kim², Dong Soon Kim², Won Dong Kim² and Sang-Bum Hong²
}

\begin{abstract}
1Department of Pulmonary and Critical Care Medicine, Ilsan Paik Hospital, Inje University, 2240, Daehwa-dong, llsanseo-gu, Goyang-si, Gyeonggido, 411-706, Korea

2Division of Pulmonary and Critical Care Medicine, Asan Medical Center, College of Medicine, University of Ulsan, 388-1, Pungnap-dong, Songpagu, Seoul, Korea
\end{abstract}

Corresponding author: Sang-Bum Hong, sbhong@amc.seoul.kr

Received: 15 Sep 2007 Revisions requested: 5 Nov 2007 Revisions received: 5 Dec 2007 Accepted: 19 Jan 2008 Published: 19 Jan 2008

Critical Care 2008, 12:R6 (doi:10.1186/cc6770)

This article is online at: http://ccforum.com/content/12/1/R6

(c) 2008 Huh et al.; licensee BioMed Central Ltd.

This is an open access article distributed under the terms of the Creative Commons Attribution License (http://creativecommons.org/licenses/by/2.0), which permits unrestricted use, distribution, and reproduction in any medium, provided the original work is properly cited.

\begin{abstract}
Background Differential diagnosis of patients with bilateral lung infiltrates remains a difficult problem for intensive care clinicians. Here we evaluate the diagnostic role of soluble triggering receptor expressed on myeloid cells-1 (sTREM-1) in bronchoalveolar lavage (BAL) specimens from patients with bilateral lung infiltrates.

Methods We conducted a prospective observational study on 80 patients with bilateral lung infiltrates with clinical suspicion of infectious pneumonia. Patients were categorized into three groups: bacterial or fungal infection, intracellular or viral infection, and noninfectious inflammatory disease. STREM-1 concentrations were measured, and BAL fluid and Clinical Pulmonary Infection Score (CPIS) were analyzed.
\end{abstract}

Results The sTREM-1 concentration was significantly increased in patients with bacterial or fungal pneumonia $(n=29$, $521.2 \pm 94.7 \mathrm{pg} / \mathrm{ml}$ ), compared with that in patients with viral pneumonia, atypical pneumonia or tuberculosis $(n=14,92.9 \pm$ $20.0 \mathrm{pg} / \mathrm{ml})$ or noninfectious inflammatory disease $(n=37,92.8$ $\pm 10.7 \mathrm{pg} / \mathrm{ml}$ ). The concentration of sTREM-1 in BAL fluid, but not CPIS, was an independent predictor of bacterial or fungal pneumonia, and a cutoff value of more than $184 \mathrm{pg} / \mathrm{ml}$ yielded a diagnostic sensitivity of $86 \%$ and a specificity of $90 \%$.

Conclusion The sTREM-1 level in BAL fluid from patients with bilateral lung infiltrates is a potential marker for the differential diagnosis of pneumonia due to extracellular bacteria.

\section{Introduction}

Differential diagnosis of patients with bilateral lung infiltrates remains a difficult problem for intensive care clinicians. Diverse presumptive clinical diagnoses of bilateral lung infiltrates include severe pneumonia induced by bacteria, virus, fungi or tuberculosis, and noninfectious inflammatory diseases caused by collagen vascular disease associated with interstitial lung disease, acute exacerbation of interstitial lung disease, pulmonary edema, acute respiratory distress syndrome or druginduced lung disease [1]. Notably, several noninfectious processes other than pneumonia lead to fever, leukocytosis, hypoxemia, purulent tracheal secretions, and diffuse pulmonary infiltrates. To enhance the specificity of clinical criteria for diagnosing ventilator-associated pneumonia, the Clinical Pulmonary Infection Score (CPIS) was introduced, which showed a high diagnostic accuracy for ventilator-associated pneumonia in some cases [2,3]. Gibot and colleagues also showed that CPIS could differentiate between patients with and without pneumonia [4]. However, the utility of CPIS remains to be validated, particularly in patients with bilateral infiltration [5]. The need for serology and microbiological tests could delay differential diagnosis for 48 to 72 hours, and the positive culture rate may be low [6-8].

$\mathrm{BAL}=$ bronchoalveolar lavage; $\mathrm{Cl}=$ confidence interval; $\mathrm{CPIS}=$ Clinical Pulmonary Infectious Score; $\mathrm{NBL}=$ non-directed bronchial lavage; ROC $=$ receiver operating characteristic; sTREM-1 = soluble triggering receptor expressed on myeloid cells-1; TREMs $=$ triggering receptors expressed on myeloid cells. 
Triggering receptors expressed on myeloid cells (TREMs) are members of the immunoglobulin (Ig) superfamily, a critical component of the innate immune defense system against infection $[9,10]$. TREM-1 expression is upregulated by extracellular bacteria and fungi but is weak in mycobacterial, viral, intracellular bacterial, and noninfectious inflammatory disorders [10-15]. However, there are conflicting reports on the potential function of soluble TREM-1 (sTREM-1) in bronchoalveolar lavage (BAL) fluid as a biomarker of ventilator-associated pneumonia measured by mini-bronchoalveolar lavage or non-directed bronchial lavage (NBL) [4,16-19]. Consequently, more clinical evidence is required to establish the diagnostic role of STREM-1 in BAL fluid. In this study we focus solely on patients with bilateral lung infiltrates, regardless of mechanical ventilation.

\section{Materials and methods Study population}

We enrolled 122 patients with bilateral lung infiltrates on the basis of clinical suspicion of infectious pneumonia, hospitalized in our medical intensive care unit between 1 April 2004 and 30 September 2005 (Figure 1) [2,20]. The study was approved by the Institutional Review Board of the Asan Medical Center, and written informed consent was obtained from patients or their relatives. Eligibility criteria included the following: (1) immunocompetent state, (2) age more than 18 years, (3) bilateral lung infiltrates on chest radiography at admission to the intensive care unit; and at least two of the following conditions: purulent sputum, temperature more than $38.3^{\circ} \mathrm{C}$ or leukocyte count of less than 4,000 or more than $11,000 / \mathrm{mm}^{3}$ (4) within 24 hours of administration of the initial antibiotic therapy or immunosuppressive therapy before BAL. In total, 42 patients were excluded because of previous treatment with nonspecific broad-spectrum antibiotics (39 patients) and an immunosuppressive state (3 patients).

\section{Figure 1}

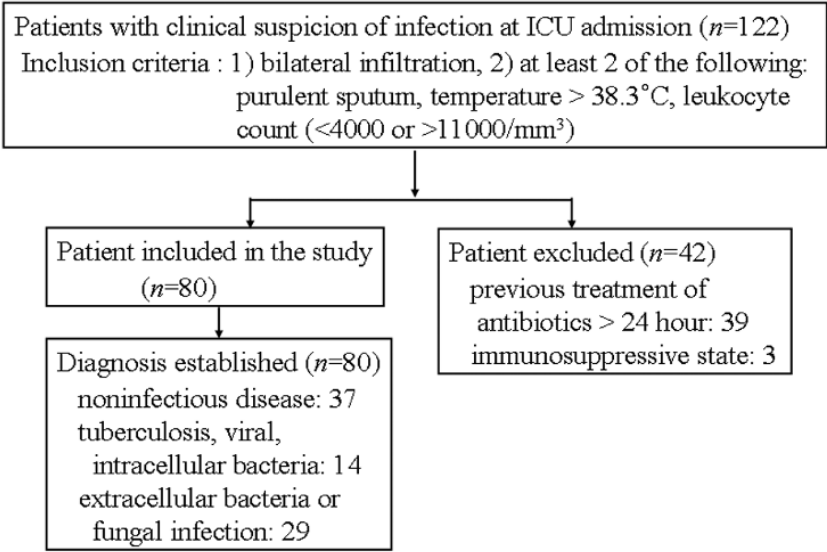

Flow diagram of patients displaying bilateral infiltration with clinical suspicion of infectious pneumonia admitted.
BAL was performed within 24 hours of admission at the intensive care unit. Additional variables recorded during admission included C-reactive protein, duration of mechanical ventilation, and length of stay in the intensive care unit. CPIS was calculated as described in a previous report [2].

Two intensivists reviewed all patient medical records and independently classified bilateral lung infiltrate diagnoses. A consensus about diagnosis was achieved in all cases. Both intensivists were unaware of the results of STREM- 1 measurements in BAL fluid. On the basis of clinical, radiological, and microbiological data, patients were assigned to one of three groups (Table 1). Group A ( $n=37$ ) consisted of patients with noninfectious diseases (for example acute exacerbation of interstitial lung disease, collagen vascular disease-associated lung disease, pulmonary edema, acute respiratory distress syndrome - excluding bacterial pneumonia or drug-induced lung disease). Group B $(n=14)$ included patients with tuberculosis, viral pneumonia, or atypical intracellular bacteria. Group C $(n=29)$ comprised patients with extracellular bacterial and fungal infections.

\section{Definition of disease}

Patients were diagnosed with non-infectious inflammatory etiology, on the basis of clinical data, radiological signs, BAL findings, and lung biopsy. The extent of interstitial lung disease exacerbation was based on the criteria of Kondoh and colleagues [21]. These conditions included: (1) aggravation of dyspnea within 1 month, (2) hypoxemia with a ratio of arterial oxygen tension to inspired oxygen tension of less than 225, (3) newly developing pulmonary infiltrates on chest radiography, and (4) absence of apparent infection or heart disease. The diagnosis of extracellular bacterial or fungal pneumonia was based on positive blood culture or quantitative culture of BAL fluid, or a rapid response of clinical symptoms and signs to antibiotic therapy. The concentration of clinically significant microorganisms for potential diagnosis of bacterial pneumonia was more than $10^{4}$ colony-forming units per $\mathrm{ml}$ of BAL fluid $[8,22,23]$. Pneumonia due to atypical intracellular bacteria (Mycoplasma pneumoniae and Legionella pneumoniae) was diagnosed on the basis of positive serologic tests showing a fourfold or greater increase in the antibody titer in paired serum samples. Diagnosis of viral pneumonia was based on clinical data, serologic tests, radiological signs [24], and biopsies.

\section{Assay of STREM-1 in bronchoalveolar lavage fluid}

Flexible bronchoscopy was performed on patients sedated with midazolam. BAL was performed either in the right middle lobe or the lingual segment by using $150 \mathrm{ml}$ of sterile physiological saline solution in three consecutive $50 \mathrm{ml}$ aliquots. The initial aspirated fluid underwent microbiological screening, and subsequent aliquots were collected for BAL analysis and sTREM-1. BAL fluid was subsequently filtered through sterile gauze to remove mucus, and then centrifuged at $500 \mathrm{~g}$ and 
Table 1

\begin{tabular}{|c|c|}
\hline Group & Diagnosis $(n)$ \\
\hline A & Acute exacerbation of interstitial lung disease (10) \\
\hline \multirow[t]{4}{*}{$(n=37)$} & Collagen vascular disease-associated lung disease ${ }^{a}(6)$ \\
\hline & Radiation pneumonitis (4) \\
\hline & Drug-induced lung disease (3) \\
\hline & Others ${ }^{b}(14)$ \\
\hline B & Atypical pneumonia (4) \\
\hline \multirow[t]{5}{*}{$(n=14)$} & Cytomegalovirus pneumonia (3) \\
\hline & Pulmonary tuberculosis (2) \\
\hline & Leptospirosis (2) \\
\hline & Pneumocystis jiroveci pneumonia (2) \\
\hline & Herpes simplex virus pneumonia (1) \\
\hline C & Bacterial pneumonia (27) \\
\hline \multirow[t]{11}{*}{$(n=29)$} & Methicillin-resistant Staphylococcus aureus (9) \\
\hline & Methicillin-susceptible Staphylococcus aureus (1) \\
\hline & Pseudomonas aeruginosa (5) \\
\hline & Klebsiella pneumoniae (2) \\
\hline & Hemophilus influenza (1) \\
\hline & ESBL K. pneumoniae (1) \\
\hline & Stenotrophomonas maltophilia (1) \\
\hline & Unknown (7) \\
\hline & Fungal pneumonia (2) \\
\hline & Candida glabrata (1) \\
\hline & Aspergillosis (1) \\
\hline
\end{tabular}

ESBL, extended-spectrum $\beta$-lactamase.

aCollagen vascular disease-associated lung disease: vasculitis $(n=$ $3)$, rheumatoid arthritis $(n=1)$, dermatomyositis $(n=1)$, and systemic lupus erythematosus $(n=1)$. bOther: acute respiratory distress syndrome $(n=3)$, malignancy-associated lung disease $(n=$ $3)$, hypersensitivity pneumonia $(n=2)$, acute eosinophilic pneumonia $(n=2)$, diffuse alveolar damage $(n=1)$, pulmonary edema $(n=1)$, sarcoidosis $(n=1)$, and postpartum hemorrhage $(n=1)$.

$4^{\circ} \mathrm{C}$ for $15 \mathrm{~min}$ to obtain the cell pellet. The supernatant was centrifuged, separated, and stored as aliquots at $-80^{\circ} \mathrm{C}$ until further analysis.

The sTREM-1 concentration in BAL fluid samples was measured with a DuoSet enzyme-linked immunosorbent assay kit (R\&D Systems, Minneapolis, MN, USA) $[16,18]$ consisting of a capture antibody (mouse anti-human TREM-1), standard antibody (recombinant human TREM-1), and detection antibody (biotinylated goat anti-human TREM-1). Intra-assay and inter-assay coefficients of variation were $2.8 \%$ and $5.2 \%$, respectively.

\section{Statistical methods}

Categorical data were compared by using Fisher's exact test, and continuous data were compared with the Kruskal-Wallis test. To evaluate the diagnostic value of data we used a logistic regression model. Receiver operating characteristic (ROC) curves were constructed to illustrate the various cutoff values of sTREM-1, CPIS, and neutrophil count in BAL fluid. Continuous variables are expressed as mean \pm SEM, and two-tailed $P$ values of less than 0.05 were considered statistically significant. All data were analyzed with SPSS version 11.0 (SPSS Inc, Chicago, IL, USA).

\section{Results \\ Patient characteristics}

Characteristics of the study subjects are shown in Table 2. Groups A and B displayed similar clinical and laboratory features. In contrast, group $C$ displayed neutrophilia in BAL fluid and a high CPIS score compared with group A (Table 3). Pathogens were cultured in $76 \%$ of samples from group $C$ patients (Table 1).

The STREM-1 concentration was significantly elevated in group $C(521.2 \pm 94.7 \mathrm{pg} / \mathrm{ml})$, compared with groups $A(92.8$ $\pm 10.7 \mathrm{pg} / \mathrm{ml}, P<0.05)$ and B $(92.9 \pm 20.0 \mathrm{pg} / \mathrm{ml}, P<0.05)$ (Figure 2). Subgroup analysis of group $\mathrm{C}$ (communityacquired pneumonia, nosocomial pneumonia, and ventilatorassociated pneumonia) disclosed that STREM-1 concentrations were not significantly different between the three subgroups (Additional File 1).

\section{Diagnostic value of the STREM-1 assay}

We employed ROC curve analysis (Figure 3) to determine whether the sTREM-1 concentration in BAL fluid can be used to discriminate between the possible causes of bilateral lung infiltrates. The area under the ROC curve, using sTREM-1 to differentiate between the presence and the absence of bacterial and fungal pneumonia, was 0.91 (95\% confidence interval (Cl) 0.83 to $0.98 ; P<0.001$ ). A sTREM-1 cutoff value of 184 $\mathrm{pg} / \mathrm{ml}$ correlated with sensitivity and specificity values of $86 \%$ (95\% Cl 72.9 to 99.6 ) and $90 \%$ (95\% Cl 81.8 to 98.7 ), respectively. A positive likelihood ratio of 8.79 , a negative likelihood ratio of 0.11 , and an odds ratio of 57.50 (95\% Cl 14.15 to 233.66) were calculated. At a level of $184 \mathrm{pg} / \mathrm{ml}$ or higher, sTREM-1 was detected in BAL fluid from 25 of 29 patients with bacterial or fungal pneumonia (sensitivity $86 \% ; 4$ falsenegative results), 4 of 37 patients with noninfectious inflammatory disease ( 4 false-positive results), and 1 of 14 patients with atypical pneumonia, viral pneumonia, or tuberculosis (1 falsepositive result). Three of the five false-positive cases showed diffuse alveolar hemorrhage in BAL fluid without reference to infection. On exclusion of patients with diffuse alveolar hemorrhage, the sTREM-1 cutoff value of $184 \mathrm{pg} / \mathrm{ml}$ yielded sensitivity and specificity values of $92 \%(95 \% \mathrm{Cl} 80.6$ to 100$)$ and $95 \%$ (95\% Cl 87.6 to 100), respectively. 
Critical Care Vol 12 No 1 Huh et al.

Table 2

\begin{tabular}{|c|c|c|c|}
\hline Characteristic & Group A $(n=37)$ & Group B $(n=14)$ & Group C $(n=29)$ \\
\hline Age, years & $57.8 \pm 2.8$ & $63.7 \pm 3.4$ & $61.7 \pm 3.3$ \\
\hline Sex, M:F & $22: 15$ & $10: 4$ & 23:6 \\
\hline APACHE II score at entry & $18.1 \pm 1.0$ & $16.8 \pm 1.4$ & $21.6 \pm 1.4^{\mathrm{a}}$ \\
\hline \multicolumn{4}{|l|}{ Co-morbidities, $n$} \\
\hline Malignancy & 12 & & 6 \\
\hline Chronic heart disease & 2 & 1 & 1 \\
\hline Chronic lung disease & & 2 & 3 \\
\hline Chronic liver disease & & 1 & 2 \\
\hline Chronic renal disease & 1 & & 1 \\
\hline Endocrinologic disease & 4 & & \\
\hline Neurologic disease & 2 & & 6 \\
\hline Transplantation & & 1 & 1 \\
\hline Duration of mechanical ventilation, days & $11.3 \pm 1.7$ & $10.2 \pm 3.6$ & $11.2 \pm 2.7$ \\
\hline Length of stay in ICU, days & $16.3 \pm 2.2$ & $10.9 \pm 3.1$ & $18.3 \pm 2.7^{a}$ \\
\hline Mortality in ICU, percentage & 40.5 & 28.6 & 42.9 \\
\hline
\end{tabular}

Results are presented as mean \pm SEM. Group A: noninfectious; group BI virus, tuberculosis, intracellular bacteria; group C: extracellular bacteria, fungi. APACHE, Acute Physiology and Chronic Health Evaluation; ICU, intensive care unit.

a $P<0.05$ versus group $B$.

A multiple logistic regression analysis showed that the sTREM-1 level (184 pg/ml) in BAL fluid is an independent predictor of bacterial or fungal pneumonia with an odds ratio of 59.742 (95\% Cl 6.610 to 539.930 ) (Table 4). No correlation was evident between the neutrophil count and STREM-1 in BAL fluid $(r=0.214, P=0.069)$.

\section{Discussion}

The main findings of this study are that sTREM-1 concentration can be used effectively in the diagnosis of bacterial or fungal pneumonia in patients with bilateral infiltration, and that a modified CPIS of more than 6 is not a valid diagnostic indicator of pneumonia using multivariate analysis.

Table 3

Characteristics of the three groups of patients with bilateral lung infiltrates at enrollment

\begin{tabular}{llll}
\hline Characteristic & Group A $(n=37)$ & Group B $(n=14)$ & Group C $(n=29)$ \\
\hline CPIS & $6.4 \pm 0.4$ & $7.4 \pm 0.4$ & $8.8 \pm 0.4^{\mathrm{a}}$ \\
C-reactive protein, mg/dl & $11.0 \pm 1.5$ & $14.0 \pm 2.0$ & $12.6 \pm 2.6$ \\
BAL fluid findings, percentage & & & $66.7 \pm 7.0^{\mathrm{a}}$ \\
$\quad$ Neutrophils & $34.6 \pm 8.6$ & $36.0 \pm 15.0$ & $15.7 \pm 4.1^{\mathrm{a}}$ \\
$\quad$ Alveolar macrophages & $29.3 \pm 8.0$ & $28.7 \pm 6.4$ & $11.2 \pm 5.3$ \\
$\quad$ Lymphocytes & $22.5 \pm 6.1$ & $24.5 \pm 10.9$ & $3.7 \pm 2.6$ \\
$\quad$ Eosinophils & $7.0 \pm 3.4$ & $8.2 \pm 5.2$ & $521.2 \pm 94.7^{\mathrm{a} b}$
\end{tabular}

Results are presented as mean \pm SEM. Group A: noninfectious; group B: virus, tuberculosis, intracellular bacteria; group C: extracellular bacteria, fungi. CPIS, Clinical Pulmonary Infection Score; BAL, bronchoalveolar lavage; sTREM-1, soluble triggering receptor expressed on myeloid cells-1. a $P<0.05$ versus group $A ;$ b $P<0.05$ versus group $B$. 
Figure 2

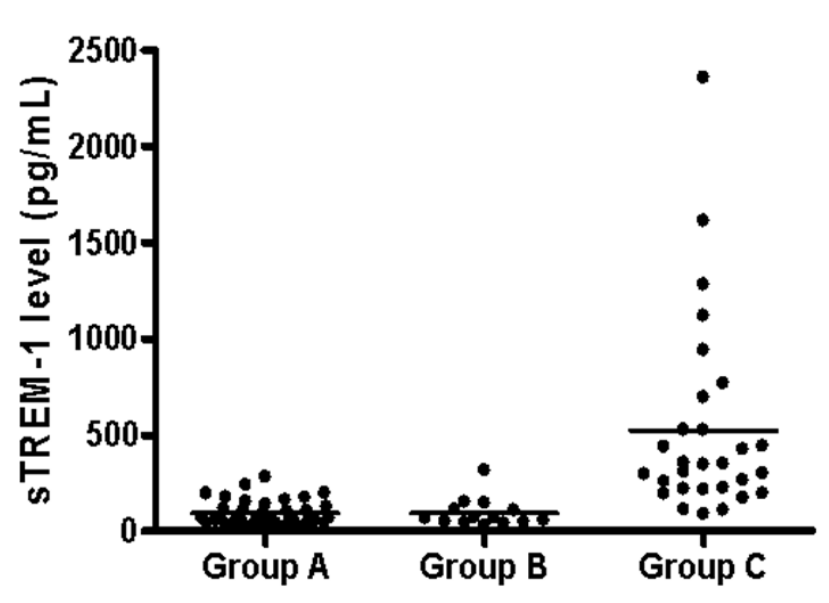

Concentration of sTREM-1 in bronchoalveolar lavage fluid of patients with bilateral lung infiltrates. Group A, noninfectious inflammatory disease; group B, atypical pneumonia, viral pneumonia, and tuberculosis; group C, bacterial or fungal pneumonia. Individual values are plotted; bars represent the median values. sTREM-1, soluble triggering receptor expressed on myeloid cells- 1 .

Figure 3

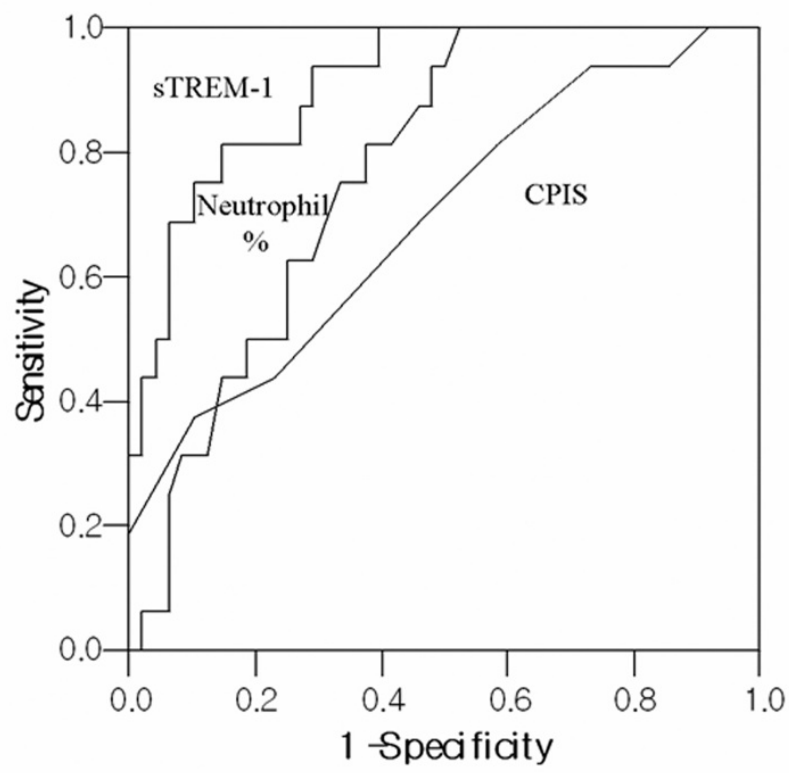

ROC curve of sTREM-1, neutrophil percentage in BAL fluid, and CPIS for diagnosis of bacterial and fungal pneumonia. Areas under the receiver operating characteristic $(\mathrm{ROC})$ curve were 0.91 (95\% confidence interval $(\mathrm{Cl}), 0.83$ to $0.98 ; P=0.000$ ) for soluble triggering receptor expressed on myeloid cells-1 (sTREM-1), 0.77 (95\% Cl 0.54 to $0.84 ; P=0.001$ ) for percentage of neutrophils in bronchoalveolar lavage fluid, and $0.69(95 \% \mathrm{Cl} 0.54$ to $0.84 ; P=0.023)$ for Clinical Pulmonary Infection Score (CPIS).
In cases where patients displayed localized consolidation on a chest radiogram, diagnosing pneumonia is less difficult than identifying the cause of bilateral infiltration. The appropriate diagnosis of bilateral lung infiltrates in critically ill patients is crucial but difficult. In many cases, bilateral lung infiltrates are associated with noninfectious inflammatory diseases. Although previous reports show that a CPIS of more than 6 indicates a high likelihood of pneumonia, its diagnostic accuracy in bilateral lung infiltrates is controversial [2,4]. A CPIS value greater than 6 was also a useful screening tool $(82 \%$ sensitivity) in the present study, but its specificity for differential diagnosis of bilateral bacterial pneumonia was low (39\%). In contrast to our findings, Gibot and colleagues reported that CPIS could be effectively applied to differentiate between patients with and without pneumonia (including communityacquired pneumonia). Our study included $68.4 \%$ of patients with a CPIS of more than 6 , compared with $49 \%$ of patients in Gigot's study. It therefore seems that a CPIS of more than 6 is not an efficient factor in the diagnosis of pneumonia with bilateral lung infiltrates.

Several earlier studies have focused on STREM-1 in patients with pneumonia. The present study, however, involved only bilateral lung infiltration and took into consideration several cases of acute exacerbation of interstitial lung disease, which is difficult to distinguish from superimposed pneumonia. Although some patients with bilateral infiltration were analyzed by Gibot and colleagues, this condition was not the focus of the earlier study. In addition, Gibot and colleagues did not include patients with acute exacerbation of idiopathic pulmonary fibrosis or viral pneumonia [4]. In another study, Richeldi and coworkers did not include patients with pneumonia caused by 'atypical' intracellular pathogens or fungi or those admitted to the intensive care unit, and employed cytofluorimetric analysis [25]. Our results not only confirm several previous findings but also provide additional information.

Here we show that the sTREM-1 level in BAL fluid constitutes an independent factor in the differential diagnosis of bacterial or fungal pneumonia at a cutoff level higher than $184 \mathrm{pg} / \mathrm{ml}$. Determann and coworkers reported that at a cutoff value of $200 \mathrm{pg} / \mathrm{ml}$, sTREM-1 levels in NBL fluid in ventilator-associated pneumonia yielded diagnostic sensitivity and specificity values of $75 \%$ and $84 \%$, respectively [16]. This study was performed with bronchoscopic BAL fluid instead of NBL fluid. Previous data were obtained primarily with NBL fluid, which may differ from BAL fluid in terms of specific characteristics. However, the sTREM-1 levels were not significantly different between BAL fluid and NBL fluid.

In the present study we observed no correlation between neutrophil counts and sTREM-1 levels in BAL fluid, indicating that activation of neutrophils and amplification of the inflammatory response occur via different mechanisms. sTREM-1 may have a role in acute inflammation characterized by an exudate of 
Table 4

Multiple logistic-regression analysis of factors used for differential diagnosis of bacterial or fungal pneumonia

\begin{tabular}{llll}
\hline Predictor & Odds ratio & $95 \% \mathrm{Cl}$ & $P$ \\
\hline BAL fluid sTREM-1 level $\geq 184 \mathrm{pg} / \mathrm{ml}$ & 59.742 & $6.610-539.930$ & 0.000 \\
BAL neutrophils $\geq 60 \%$ & 11.517 & $1.227-108.084$ & 0.032 \\
CPIS $>6$ & 0.484 & $0.068-3.459$ & 0.470 \\
\hline
\end{tabular}

BAL, bronchoalveolar lavage; sTREM-1, soluble triggering receptor expressed on myeloid cells-1; CPIS, Clinical Pulmonary Infection Score; Cl, confidence interval.

neutrophils and monocytes. Moreover, lipopolysaccharides, bacteria, and fungi upregulate sTREM-1 expression [10,1215].

The present study has several limitations. First, because most of the false-positive results in STREM-1 levels involved diffuse alveolar hemorrhage, which was not included in other investigations $[4,16,18,25]$, the utility of STREM-1 in this group remains to be determined. Second, some patients may have suffered from noninfectious inflammatory disease combined with infection, although two blinded investigators determined each patient's diagnosis without knowledge of the sTREM-1 concentration. Third, the sTREM level measured in BAL fluid is lower as a result of dilution and may differ from the actual concentrations in some patients, although we performed exactly the same technique and retrieved similar volumes in the three groups (data not shown). Finally, cases of fungal pneumonia were rare.

\section{Conclusion}

The sTREM-1 level in BAL fluid from patients with bilateral lung infiltrates is a potential marker for the differential diagnosis of pneumonia due to extracellular bacteria. We propose that the sTREM-1 level (184 pg/ml or more, versus less than $184 \mathrm{pg} /$ $\mathrm{ml}$ ) is a more useful marker than clinical criteria in refining the diagnostic spectrum (bacterial infection versus others) in patients presenting bilateral lung infiltrates.

\section{Key messages \\ - The sTREM-1 concentration in BAL fluid is an inde- pendent predictor of bacterial or fungal pneumonia in patients with bilateral lung infiltrates, and a cutoff value of more than $184 \mathrm{pg} / \mathrm{ml}$ yields a diagnostic sensitivity of $86 \%$ and a specificity of $90 \%$. \\ - A modified Clinical Pulmonary Infection Score of more than 6 does not show clinical usefulness for the diagno- sis of pneumonia in patients with bilateral lung infiltrates. \\ - The sTREM-1 level may be applied as a useful marker for the differential diagnosis of bilateral lung infiltrates.}

\section{Competing interests}

The authors declare that they have no competing interests.

\section{Authors' contributions}

HJW and HSB initiated the study. KYS, LCM, OYM, STS, LSD, KWS, KDS, and KWD participated in patient management. HJW and HSB analyzed the data. All the authors contributed to and approved the final manuscript.

\section{Additional files}

The following Additional files are available online:

\section{Additional file 1}

file containing two supplementary tables.

See http://www.biomedcentral.com/content/ supplementary/cc6770-S1.doc

\section{Acknowledgements}

We thank Eun-Mi Cho for help with clinical duties, and Eun-Mi Park for technical assistance. This work was supported by the Asan Institute for Life Science (grant no. 2005-375).

\section{References}

1. Butler KL, Sinclair KE, Henderson VJ, McKinney G, Mesidor DA, Katon-Benitez I, Weaver WL: The chest radiograph in critically ill surgical patients is inaccurate in predicting ventilator-associated pneumonia. Am Surg 1999, 65:805-809.

2. Pugin J, Auckenthaler R, Mili N, Janssens JP, Lew PD, Suter PM: Diagnosis of ventilator-associated pneumonia by bacteriologic analysis of bronchoscopic and nonbronchoscopic 'blind' bronchoalveolar lavage fluid. Am Rev Respir Dis 1991, 143:1121-1129.

3. Fartoukh M, Maitre B, Honore S, Cerf C, Zahar JR, Brun-Buisson $\mathrm{C}$ : Diagnosing pneumonia during mechanical ventilation: the clinical pulmonary infection score revisited. $A m J$ Respir Crit Care Med 2003, 168:173-179.

4. Gibot S, Cravoisy A, Levy B, Bene MC, Faure G, Bollaert PE: Soluble triggering receptor expressed on myeloid cells and the diagnosis of pneumonia. N Engl J Med 2004, 350:451-458.

5. Chastre J, Fagon JY: Ventilator-associated pneumonia. $A m \mathrm{~J}$ Respir Crit Care Med 2002, 165:867-903.

6. Meduri GU, Wunderink RG, Leeper KV, Beals DH: Management of bacterial pneumonia in ventilated patients. Protected bronchoalveolar lavage as a diagnostic tool. Chest 1992, 101:500-508.

7. Papazian L, Thomas P, Garbe L, Guignon I, Thirion X, Charrel J, Bollet C, Fuentes P, Gouin F: Bronchoscopic or blind sampling techniques for the diagnosis of ventilator-associated pneumonia. Am J Respir Crit Care Med 1995, 152:1982-1991.

8. Kollef $M H$, Bock KR, Richards RD, Hearns ML: The safety and diagnostic accuracy of minibronchoalveolar lavage in patients with suspected ventilator-associated pneumonia. Ann Intern Med 1995, 122:743-748. 
9. Zhang P, Summer WR, Bagby GJ, Nelson S: Innate immunity and pulmonary host defense. Immunol Rev 2000, 173:39-51.

10. Bouchon A, Dietrich J, Colonna M: Cutting edge: inflammatory responses can be triggered by TREM-1, a novel receptor expressed on neutrophils and monocytes. J Immunol 2000, 164:4991-4995.

11. Colonna M, Facchetti F: TREM-1 (triggering receptor expressed on myeloid cells): a new player in acute inflammatory responses. J Infect Dis 2003, 187(Suppl 2):S397-S401.

12. Bleharski JR, Kiessler V, Buonsanti C, Sieling PA, Stenger S, Colonna M, Modlin RL: A role for triggering receptor expressed on myeloid cells-1 in host defense during the early-induced and adaptive phases of the immune response. J Immunol 2003, 170:3812-3818.

13. Bouchon A, Facchetti F, Weigand MA, Colonna M: TREM-1 amplifies inflammation and is a crucial mediator of septic shock. Nature 2001, 410:1103-1107.

14. Nathan C, Ding A: TREM-1: a new regulator of innate immunity in sepsis syndrome. Nat Med 2001, 7:530-532.

15. Cohen J: TREM-1 in sepsis. Lancet 2001, 358:776-778.

16. Determann RM, Millo JL, Gibot S, Korevaar JC, Vroom MB, van der Poll T, Garrard CS, Schultz MJ: Serial changes in soluble triggering receptor expressed on myeloid cells in the lung during development of ventilator-associated pneumonia. Intensive Care Med 2005, 31:1495-1500.

17. Gibot S, Kolopp-Sarda MN, Bene MC, Cravoisy A, Levy B, Faure GC, Bollaert PE: Plasma level of a triggering receptor expressed on myeloid cells-1: its diagnostic accuracy in patients with suspected sepsis. Ann Intern Med 2004, 141:9-15.

18. Horonenko G, Hoyt JC, Robbins RA, Singarajah CU, Umar A, Pattengill J, Hayden JM: Soluble triggering receptor expressed on myeloid cell-1 is increased in patients with ventilator-associated pneumonia: a preliminary report. Chest 2007, 132:58-63.

19. Gibot S, Cravoisy A, Dupays R, Barraud D, Nace L, Levy B, Bollaert PE: Combined measurement of procalcitonin and soluble TREM-1 in the diagnosis of nosocomial sepsis. Scand J Infect Dis 2007, 39:604-608.

20. Andrews CP, Coalson JJ, Smith JD, Johanson WG Jr: Diagnosis of nosocomial bacterial pneumonia in acute, diffuse lung injury. Chest 1981, 80:254-258.

21. Kondoh $Y$, Taniguchi H, Kawabata $Y$, Yokoi T, Suzuki K, Takagi K: Acute exacerbation in idiopathic pulmonary fibrosis. Analysis of clinical and pathologic findings in three cases. Chest 1993 , 103:1808-1812.

22. Souweine B, Veber B, Bedos JP, Gachot B, Dombret MC, Regnier $B$, Wolff M: Diagnostic accuracy of protected specimen brush and bronchoalveolar lavage in nosocomial pneumonia: impact of previous antimicrobial treatments. Crit Care Med 1998, 26:236-244.

23. Michel F, Franceschini B, Berger $P$, Arnal JM, Gainnier M, Sainty JM, Papazian L: Early antibiotic treatment for BAL-confirmed ventilator-associated pneumonia: a role for routine endotracheal aspirate cultures. Chest 2005, 127:589-597.

24. Moreno L, Krishnan JA, Duran P, Ferrero F: Development and validation of a clinical prediction rule to distinguish bacterial from viral pneumonia in children. Pediatr Pulmonol 2006, 41:331-337.

25. Richeldi L, Mariani M, Losi M, Maselli F, Corbetta L, Buonsanti C, Colonna M, Sinigaglia F, Panina-Bordignon P, Fabbri LM: Triggering receptor expressed on myeloid cells: role in the diagnosis of lung infections. Eur Respir J 2004, 24:247-250. 